\title{
Reliability Modeling and Analysis of One-Shot Systems
}

\author{
Dhominick Pagulayan, Jang-wook Hur, Kyeong-Hwan Hwang, Bo-Hyeon Kim
}

\begin{abstract}
The actual reliability of one-shot systems such as missiles can only be determined once they have been used up, therefore, estimating the reliability of such system requires a lot of investigation. One-shot systems spend most of their life in dormant storage where numerous tests are implemented meaning these systems are highly reliable in nature because a failure in tests leads to zero reliability which is not interesting. The challenge is to estimate the reliability of one-shot systems even if few or no failures are observed. This paper summarizes several one-shot system reliability modeling and analysis to improve the understanding of reliability engineers in estimating the reliability of these systems
\end{abstract}

\section{Keywords—Field Data, Reliability, Weibull Distribution}

\section{INTRODUCTION}

Most reliability papers discuss in detail the different methods of determining the reliability of continuous-use systems as well as intermittent-use systems but these models used in estimating the reliability of such systems cannot be applied to one-shot systems which are also called impulse systems. To illustrate clearly why methods in predicting the reliability of continuous-use systems cannot be used for oneshot systems, the assumptions regarding between the two systems will be compared.

The reliability of continuous-use systems is described as the number of failures happening over a period of time which is usually described as mean time between failures (MTBF). For one-shot systems, the failure can only happen once, although, there are times wherein the one-shot system fails a test during its dormant period which is not part of this paper because the reliability of those systems is quite low which does not need any modeling. Another problem with reliability models of continuous-use systems is that the reliability of such systems are measured during their operating time because most failures occurring as a result of the system reaching its MTBF. Unlike one-shot systems like missiles, when delivered to a specified target, does not have time to be repaired if it fails during its flight [1]. Furthermore, continuous-use systems, in general, fail because of the operation of the system whereas one-shot

Dhominick Pagulayan, Kumoh National Institute of Technology, South Korea.dapagulayan17@yahoo.com

Jang-wook Hur, Kumoh National Institute of Technology, South Korea hhjw88@gmail.com

Kyeong-Hwan Hwang, Kumoh National Institute of Technology, South Korea hkhlove89@gmail.com

Bo-Hyeon Kim, Kumoh National Institute of Technology, South Korea. koc343434@gmail.com system failures are dominated by birth defects and timedependent defects. Birth defects, which is common at the beginning of life of the system, are flaws of the system which were not identified during the product acceptance stage can be fixed after tests have identified these flaws and at the end of life of the one-shot system, time-dependent defects start to affect the reliability of the system more than the birth defects because the former is growing.

\section{CONCEPTUAL MODEL OF RELIABILITY}

Several models have been used in modeling the reliability of one-shot systems. For instance, the reliability of the oneshot systems like nuclear weapons was incorrectly modeled as the bathtub curve which was pointed out by reference [2]. In order to correctly model the reliability of one-shot systems, the concept of actual reliability and estimated reliability should be well understood first by the reliability engineers dealing with these types of systems. Note that the system, from now on, refer to one-shot systems unless specified otherwise.

\section{A. Actual Reliability Model}

The actual reliability of one-shot systems cannot be measured because it is the natural reliability of the system. They can only be theoretically visualized and cannot be determined until the use of the one-shot system. The importance of the actual reliability is that it can be inferred when there are changes that are happening in the system.

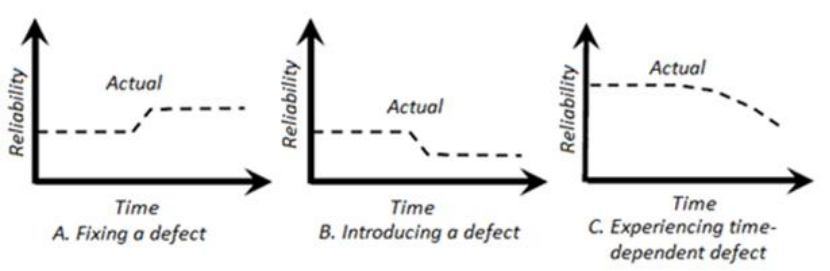

Fig. 1 Changes in actual reliability of a system

The first graph (A) shows that the actual reliability of the system will increase when a defect is found and fixed. The slope of the change in reliability of the system entirely depends on how significant the defect is to the system. In general, the first few tests reveal the critical defects thus the change in reliability of the system after fixing these defects is bigger compared to succeeding fixes. The next graph (B) shows that reliability will decrease when a defect is introduced to the system. These defects are obviously undesirable but they cannot be prevented from happening because they are accidental mistakes. An example would be when a fix is not 
correctly implemented hence affecting the reliability of other components to decrease or when an upgrade is carried out and because of unforeseen circumstances, the reliability of the system also decreases. This is important to note because making changes to the system might induce a defect thus changes must be monitored so that their impact on the reliability of the system is positive. The last graph (C) shows that a time-dependent defect is growing which is lowering the reliability of the system. These defects are usually because of environmental stimuli such as vibration, temperature, humidity, etc. and component aging. These factors must be considered in determining the end-of-life of the one-shot systems

\section{B. Estimated Reliability}

Estimating the reliability of the system is the most challenging part of being a reliability engineer because this will ultimately decide if the system will fail or not when the system is needed to be deployed. Although high estimated reliability of the system is practically good, the reliability of the system essentially dictates failure or not. This means that the error between the estimated reliability and actual reliability is as important as increasing the reliability of the system.

\section{Relationship of Actual and Estimated Reliability}

In this section, the different factors affecting how the actual and estimated reliability is discussed. Normally, the actual reliability of the system would be lower than the estimated reliability to because the assumption is that there are no defects present in the system even though there are defects present but since the tests do not show the defect, it is assumed that they are not in the system.
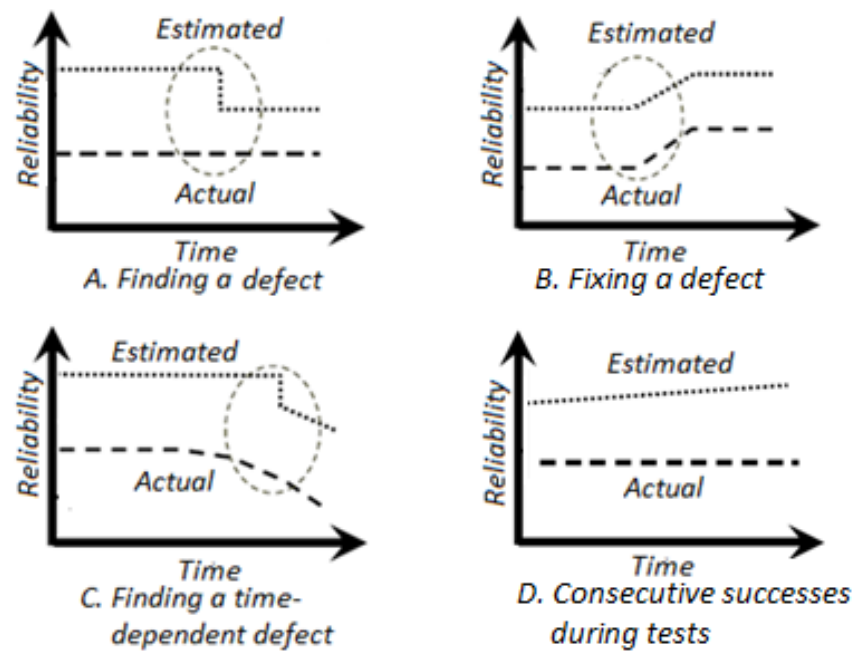

Fig. 2 Relationship of actual and estimated reliability

Finding a defect, as shown in Figure 2A, will decrease the estimated reliability because a birth defect is detected while the actual reliability does not change because the defect is still not fixed. When a defect is found, the estimated reliability improves because it approaches the actual reliability of the system. After the birth has been fixed the both estimated and actual reliability of the system increases which is illustrated in Figure 2B. As time goes on, the reliability of the components of the system deteriorates as seen from the actual reliability of the system in Figure $2 \mathrm{C}$ and by doing tests, the estimated reliability can converge to the actual reliability when tests are done. Lastly, when testing of the system reveals no failure, the estimated reliability of the system increases even though the actual reliability is not changing (see Figure 2D). Successes achieved during the testing of the system does not certainly tell that the reliability of the system is high, it might also tell that the testing program is not adequate.

\section{Reducing the Error between Actual and Estimated}

In the previous section, the more successful tests can actually be more dangerous in estimating the actual reliability of the system when the estimate for the reliability is greater than the actual reliability of the system. In order to prevent a discrepancy in estimating the reliability of the system, it can be assumed that defects are present in the system, therefore, lowering the estimate of the reliability which is better than a higher reliability estimate with a lower actual reliability. Also, this enables more tests to be more effective not only in detecting defects but also improve the estimate.
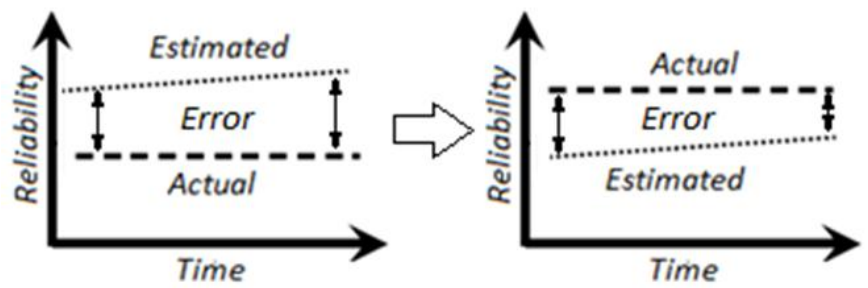

Fig. 3 Reducing level of confidence of the estimated reliability

\section{Method Of ESTIMATING RELIABILITY}

Different methods have been used in estimating the reliability of one-shot systems. The simplest method is using the normal distribution approximation but can only be used where the probability of failure is in the middle. A notable method in approximating the reliability of components of a system is the beta-binomial lower bound which is:

$$
\begin{gathered}
1-C L=P(X \leq y)=\sum_{i=0}^{y}\left(\begin{array}{l}
n \\
i
\end{array}\right) r^{i}(1-r)^{n-i} \\
P(X \leq y)=r^{n_{x}} \text { if } y=0
\end{gathered}
$$

where $\mathrm{P}(\mathrm{X}<\mathrm{y})$ is the confidence level or probability that the number of successes $X$ is greater than the number of failures $y$; $r$ is the reliability of the system and; $n$ is the number of tests done on the components. It can be noted here that (2) will be used when there are no failures and (1) otherwise. By using (2) above, reference [3] were able to get the mean and variance of the reliability of the component following the beta-binomial distribution which are:

$$
\begin{aligned}
& E[r]=\frac{n}{n+1}, \quad \text { and } \\
& \operatorname{var}(r)=\frac{n}{(n+1)^{2}(n+2)}
\end{aligned}
$$

where $E[r]$ is the mean reliability and $\operatorname{var}(r)$ is the variance of the distribution. Note that these equations can only be used when there are no failures in component testing. If failures are present, equation (1) is used to define the probability distribution to approximate the mean and variance of the 
sample.

\section{A. Procedure in Estimating Reliability of One-Shot Systems}

After computing for the mean and variance of the reliability of each component in the system, the overall system mean and variance of reliability can be computed by applying parallel and series system analysis which is discussed in detail in the next sections [4]. To get the probability distribution of the reliability of the system, the following equations are used to get parameters $\alpha$ and $\beta$ :

$$
\begin{gathered}
\alpha=E\left(r_{g}\right)\left(\frac{E^{2}\left(r_{s}\right)-E^{2}\left(r_{g}\right)}{\operatorname{Var}\left(r_{s}\right)}-1\right) \\
\beta=\left(1-E\left(r_{g}\right)\right)\left(\frac{E\left(r_{s}\right)-E^{2}\left(r_{g}\right)}{\operatorname{Var}\left(r_{s}\right)}-1\right)
\end{gathered}
$$

where $r_{s}$ is the reliability of the system. These parameters are to be used in a beta distribution $\beta\left(r_{s} ; \alpha, \beta\right)$ which is used to determine the confidence level needed by system requirements. To visualize the method clearly, see chapter 4 where an example is demonstrated.

\section{B. Series System}

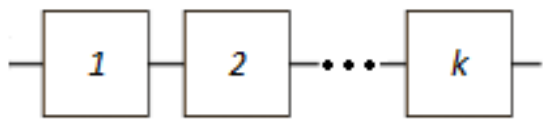

Fig. 4 Series system

The reliability of a system with components in series connections can be computed using the equation of mean and variance proposed by reference [5] which approximates the probability distribution of the system:

$$
\begin{gathered}
\mathrm{E}\left(\mathrm{r}_{g}\right)=\prod_{\mathrm{i}=1}^{k} \frac{\mathrm{n}_{\mathrm{i}}}{\mathrm{n}_{\mathrm{i}}+1} \\
\operatorname{Var}\left(\mathrm{r}_{g}\right)=\mathrm{E}\left(\mathrm{r}_{g}\right) \prod_{i=1}^{k} \frac{n_{\mathrm{i}}+1}{n_{\mathrm{i}}+2}-\mathrm{E}^{2}\left(\mathrm{r}_{g}\right)
\end{gathered}
$$

where $n_{i}$ is the number of units tested for the $i^{\text {th }}$ component. The above equation assumes that there are no failures that happened during component testing.

\section{C.Parallel System}

The reliability of a system with components in parallel was also from derived from the assumption that there were no failures in component testing. The mean and variance of the system can be computed as:

$$
\begin{gathered}
\mathrm{E}\left(\mathrm{r}_{\mathrm{p}}\right)=1-\prod_{i=1}^{k} \frac{1}{n_{\mathrm{i}}+1} \\
\operatorname{Var}\left(\mathrm{r}_{g}\right)=\left[1-\mathrm{E}\left(\mathrm{r}_{\mathrm{p}}\right)\right]\left[\prod_{\mathrm{i}=1}^{k} \frac{2}{n_{\mathrm{i}}+2}-1+\mathrm{E}\left(\mathrm{r}_{p}\right)\right]
\end{gathered}
$$

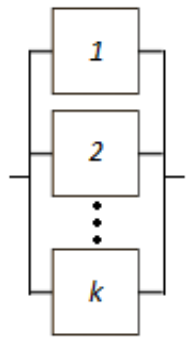

Fig. 5 Parallel system

\section{Complex System}

Complex systems are just a combination of series and parallel subsystems which can be simplified as one whole system by using equations 7 and 8 for subsystems connected in series or equations 9 and 10 for subsystems connected in parallel. A complex system is given below as an example that is to be analyzed in the next chapter.

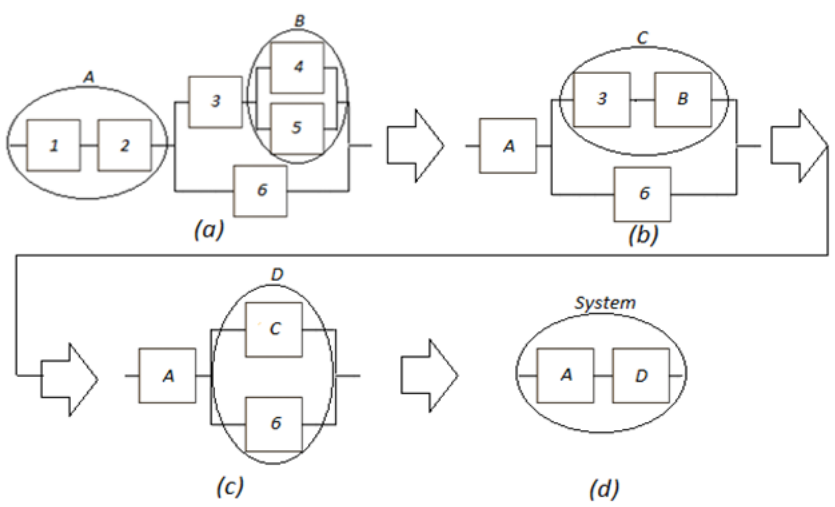

Fig. 6 Reduction of a complex system into simpler modules

As can be seen from Figure 6a, components 1 and 2 are connected in series thus can be simplified as module A while components 4 and 5 are connected in parallel thus can be simplified as module B. The original system can be drawn just like in Figure 6a. The simplified system can be further reduced to Figure $6 \mathrm{c}$ because the component 3 and module $\mathrm{B}$ are in series thus can be called module C. Subsequently, module D and component 6 are parallel simplifying them together into module D. Finally, the system is made up of two modules connected in series. When combining modules, the following equations must be used in estimating the mean and variance of complex system:

$$
\begin{gathered}
\mathrm{E}\left(\mathrm{r}_{g}\right)=\prod_{i=1}^{k} r_{\mathrm{i}}^{\mathrm{i}} \\
\operatorname{Var}\left(\mathrm{r}_{\mathrm{g}}\right)=\prod_{\mathrm{i}=1}^{k} r_{\mathrm{i}}^{2}-\prod_{\mathrm{i}=1}^{k}\left(r_{\mathrm{i}}^{2}-\operatorname{var}\left(r_{\mathrm{i}}\right)\right) \\
\mathrm{E}\left(\mathrm{r}_{\mathrm{p}}\right)=1-\prod_{i=1}^{k}\left(1-r_{\mathrm{i}}\right) \\
\operatorname{Var}\left(\mathrm{r}_{\mathrm{p}}\right)=\prod_{i=1}^{k}\left(\left(1-r_{\mathrm{i}}\right)^{2}+\operatorname{var}\left(r_{\mathrm{i}}\right)\right)-\prod_{i=1}^{k}\left(1-r_{\mathrm{i}}\right)^{2}
\end{gathered}
$$

\section{E. Network System}

Some systems are not easily reducible into a single system just like complex systems. These systems are called network 
systems because they work together and simplifying a part of the network system will lead to a different system. In solving network systems, the flow of how the components are analyzed to produce a reducible system just like a complex system. Figure 7 illustrates how network systems are analyzed.
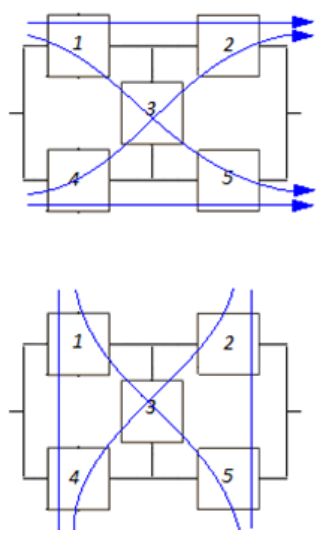

(A)
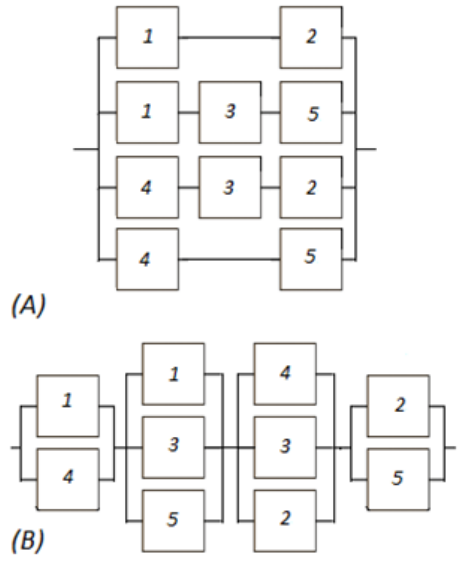

Fig. 7 Network system analysis [6]

From the figure 7, it can be observed that a network system can be interpreted into two complex systems. Figure 7A shows that components 1 and 2 can be interpreted as series connection thus making the two parallel with the other components connected in series. Figure $7 \mathrm{~B}$ shows that the same system can be interpreted as components 1 and 4 connected in parallel thus making them connected in series with the other components connected in parallel. Both models show different systems thus it is critical to choose which of the two is to be used but it is highly recommended to simulate both systems to be confident in the model.

\section{EXAMPLE SimULATION}

For a confidence level of $90 \%$, calculate the reliability of the one-shot system (Figure 6) using the component data given in Table 1. Also, calculate the number of successful trials needed to have a reliability of 0.95 with $90 \%$ confidence level with and without using the component data as a prior.

TABLE I

COMPONENT DATA OF SYSTEM IN FIGURE 6

\begin{tabular}{|c|c|c|c|c|}
\hline $\mathrm{i}$ & $\mathrm{n}_{\mathrm{i}}$ & $\mathrm{y}_{\mathrm{i}}$ & $\mathrm{r}_{\mathrm{i}}$ & $\operatorname{Var}\left(\mathrm{r}_{\mathrm{i}}\right)$ \\
\hline 1 & 15 & 0 & 0.9375 & $3.447 \times 10^{-3}$ \\
\hline 2 & 15 & 0 & 0.9375 & $3.447 \times 10^{-3}$ \\
\hline 3 & 20 & 0 & 0.9524 & $2.061 \times 10^{-3}$ \\
\hline 4 & 25 & 0 & 0.9615 & $1.370 \times 10^{-3}$ \\
\hline 5 & 25 & 0 & 0.9615 & $1.370 \times 10^{-3}$ \\
\hline 6 & 20 & 0 & 0.9524 & $2.061 \times 10^{-3}$ \\
\hline
\end{tabular}

Using the concept of complex systems, the system can be simplified into different modules which are summarized in Table 2 below. Note that the equations 11 and 12 are used for modules in series while equations 13 and 14 are used for parallel modules.
TABLE II

MEAN AND VARIANCE OF THE SimPLIFIED MOdULES

\begin{tabular}{|c|c|c|c|}
\hline Step & Module & $r_{i}$ & $\operatorname{Var}\left(r_{i}\right)$ \\
\hline \multirow{2}{*}{1} & $\mathrm{~A}$ & 0.8789 & $6.071 \times 10^{-3}$ \\
\cline { 2 - 4 } & $\mathrm{B}$ & 0.9985 & $5.929 \times 10^{-6}$ \\
\hline 2 & $\mathrm{C}$ & 0.9509 & $2.061 \times 10^{-3}$ \\
\hline 3 & $\mathrm{D}$ & 0.9977 & $1.387 \times 10^{-5}$ \\
\hline 5 & System & 0.8769 & $0.006 \times 10^{-3}$ \\
\hline$\alpha$ & 14.82731 & $\beta$ & 2.08235 \\
\hline
\end{tabular}

The probability distribution can be approximated by computing the parameters of beta distribution ( $\alpha$ and $\beta$ ) using equations 5 and 6 . The probability distribution is approximated as $\beta(r ; 14.82731,2.08235)$. Figure 8 shows the cumulative probability distribution of the reliability of the system where the reliability at $90 \%$ confidence level is estimated using interpolation.

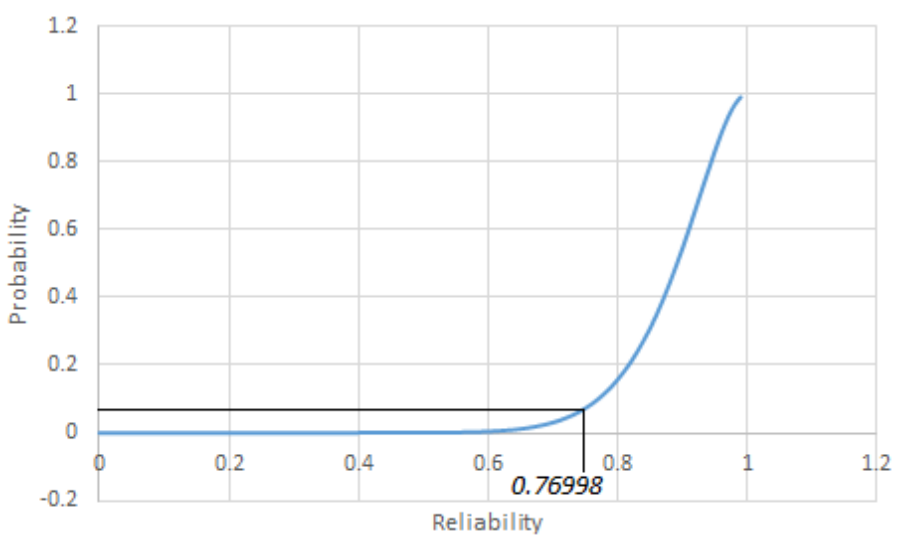

Fig. 8 Cumulative probability distribution of system

The reliability of the system at $90 \%$ confidence is predicted to be 0.76998 which can be verified by simulating it in Microsoft excel where the reliability of the components follow equation 2 and system reliability data is arranged consecutively. The number on the 1000th place $(0.76941)$ is considered the reliability at $90 \%$ confidence because there were 10,000 data points simulated. The number of trials needed for the system to achieve 0.95 reliability with $90 \%$ confidence without prior component can be computed using equation 2 thus getting $n=45$. Using the component data, the solution can be solved using the beta approximation accounting to additional successful tests: $\beta(0.95,14.82731+n$, 2.08235) where $\mathrm{n}$ can be solved and is equal to 63 which means that the system reliability is lowered by the component reliability data.

Additionally, the system reliability of one-shot systems with component testing failure can be obtained by using Equation 1 to model the probability distribution of the components and approximate the mean and variance using:

$$
\begin{gathered}
E(r)=\sum_{i=0}^{D} r * P(r), \text { and } \\
\operatorname{var}(r)=\sum_{i=0}^{D} r^{2} * P(r)-E(r)^{2}
\end{gathered}
$$


where $r$ is the reliability and $\mathrm{P}(\mathrm{r})$ is the probability that system's reliability is $r$. These equations can be used if the probability function is discretized.

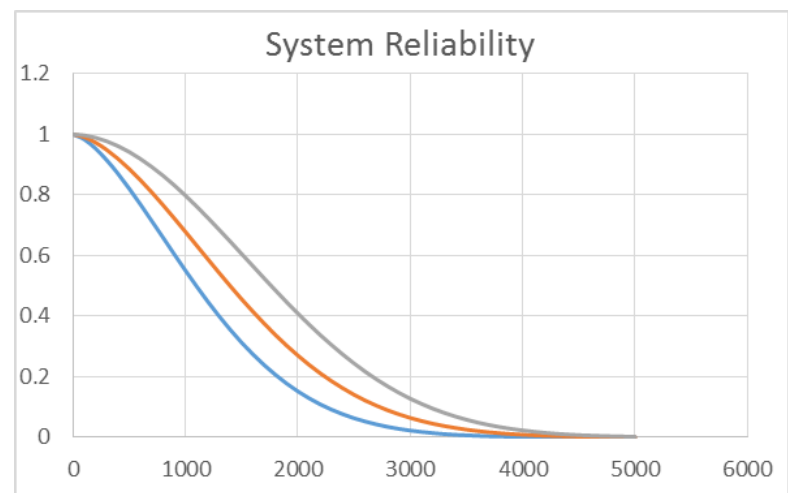

Fig. 7 System level reliability

\section{V.CONCLUSION}

Estimating the reliability of one-shot systems conceptually does not necessarily need more tests. Successful testing will probably increase the estimated reliability of the system but will diverge with the actual reliability of the system if the estimated reliability is formulated using a high confidence level, therefore, estimation must always be more conservative to account for defects not identified during testing. The method of estimating the reliability of one-shot systems might be difficult to compute but with the use of the right program, estimating the reliability will be easier. Also, using prior data in estimating how many tests should be conducted will either reduce or increase the tests needed to prove that the system has the desired level of the reliability which can either reduce the cost of tests or suggest to perform more component testing rather than system testing to achieve the desired level of reliability of the system.

\section{ACKNOWLEDGMENT}

This paper is the result of the research carried out with the support of DAPA (Defense Acquisition Program Administration, 2015-302-002).

\section{REFERENCES}

[1] Bierbaum, R., \& Wright, D. (2002). Reliability assessment methodology for 1-shot systems. Annual Reliability and Maintainability Symposium. 2002 Proceedings (Cat. No.02CH37318), 536-541. https://doi.org/10.1109/RAMS.2002.981699

[2] Bierbaum, R. L. (2010). A conceptual model for "inherent reliability" for nuclear weapons. 2010 Proceedings - Annual Reliability and Maintainability Symposium (RAMS). https://doi.org/10.1109/RAMS.2010.5447992

[3] Guo, H., Honecker, S., Mettas, A., \& Ogden, D. (2010). Reliability estimation for one-shot systems with zero component test failures. 2010 Proceedings - Annual Reliability and Maintainability Symposium (RAMS) https://doi.org/10.1109/RAMS.2010.5448016

[4] Guo, H., Jin, T., \& Mettas, A. (2011). Designing Reliability Demonstration Tests for One-Shot Systems Under Zero Component Failures. IEEE Transactions on Reliability IEEE. No. 1, 2011, pp. 286294.
https://doi.org/10.1109/TR.2010.2085552

[5] Thompson, W. E., \& Haynes, R. D. (1980). On the reliability, availability and Bayes confidence intervals for multicomponent systems. Naval Research Logistics Quarterly Naval Research Logistics, 27(3), 345-358 https://doi.org/10.1002/nav.3800270302

[6] Carlo, F. D. (2013). Reliability and Maintainability in Operations Management. INTECH Open Access Publisher. 